\title{
Construção e validação da cartilha educativa para enfermeiros sobre lesão por
}

\author{
pressão \\ Construction and validation of the educational booklet for nurses about pressure injuries \\ Construcción y validación del folleto educativo para enfermeras sobre lesiones por presión
}

Recebido: 12/02/2021 | Revisado: 20/02/2021 | Aceito: 24/02/2021 | Publicado: 04/03/2021

Lívia Bertasso Araújo Portugal

ORCID: https://orcid.org/0000-0003-3312-8871

Universidade Federal Fluminense, Brasil

E-mail: lilicabertasso@gmail.com

Bárbara Pompeu Christovam

ORCID: https://orcid.org/0000-0002-9135-8379

Universidade Federal Fluminense, Brasil

E-mail: barbarachristovam@id.uff.bb

Bernadete Lourdes Oliveira Da Silva Almeida

ORCID: https://orcid.org/0000-0001-5725-1765

Universidade Federal Fluminense, Brasil

E-mail: bealmeida1962@gmail.com

\begin{abstract}
Resumo
Objetivo: construir e validar uma cartilha educativa para enfermeiros sobre lesão por pressão. Método: estudo metodológico, desenvolvido em três etapas - revisão integrativa, elaboração de cartilha, e validação por especialistas no assunto e representantes do público-alvo. Realizou-se a validação por 8 juízes e 39 representantes do público-alvo. Considerou-se o Índice de Validade de Conteúdo mínimo de 0,80, para validação de conteúdo e concordância mínima de 75\% para validação de aparência. Resultados: A cartilha apresentou Índice de Validade de Conteúdo global de 0,90 pelos juízes e nível de concordância excelente entre os juízes (87\%-100\%) e representantes do público-alvo (96\%$100 \%$ ). Entretanto, os juízes propuseram sugestões de melhorias da cartilha, que foram acatadas e modificadas para versão final do material. Conclusão: a cartilha educativa foi validada quanto ao conteúdo e aparência, devendo ser considerada no contexto das atividades educativas como instrumento capaz de favorecer os enfermeiros na prevenção e tratamento da lesão por pressão.
\end{abstract}

Palavras-chave: Lesão por pressão; Estudo de validação; Cuidados de enfermagem; Protocolos; Enfermagem.

\begin{abstract}
Objective: to build and validate an educational booklet for nurses about pressure injuries. Method: methodological study, developed in three stages - integrative review, preparation of booklet, and validation by specialists in the subject and representatives of the target audience. Validation was carried out by 8 judges and 39 representatives of the target audience. The Minimum Content Validity Index of 0.80 was considered for content validation and minimum agreement of $75 \%$ for appearance validation. Results: The booklet presented a global Content Validity Index of 0.90 by the judges and an excellent level of agreement between the judges $(87 \%-100 \%)$ and representatives of the target audience $(96 \%-100 \%)$. However, the judges proposed suggestions for improving the booklet, which were accepted and modified for the final version of the material. Conclusion: the educational booklet was validated in terms of content and appearance, and should be considered in the context of educational activities as an instrument capable of favoring nurses in the prevention and treatment of pressure injuries.
\end{abstract}

Keywords: Pressure injury; Validation study; Nursing care; Protocols; Nursing.

\section{Resumen}

Objetivo: construir y validar un folleto educativo para enfermeras sobre lesiones por presión. Método: estudio metodológico, desarrollado en tres etapas: revisión integradora, elaboración de cuadernillo y validación por especialistas en el tema y representantes del público objetivo. La validación fue realizada por 8 jueces y 39 representantes del público objetivo. Se consideró el índice de validez de contenido mínimo de 0,80 para la validación de contenido y el acuerdo mínimo de $75 \%$ para la validación de apariencia. Resultados: El folleto presentó un índice de validez de contenido global de 0.90 por parte de los jueces y un excelente nivel de acuerdo entre los jueces ( $87 \%$ $100 \%$ ) y los representantes de la audiencia objetivo (96\% -100\%). Sin embargo, los jueces propusieron sugerencias para mejorar el folleto, que fueron aceptadas y modificadas para la versión final del material. Conclusión: el folleto educativo fue validado en términos de contenido y apariencia, y debe ser considerado en el contexto de las actividades 
educativas como un instrumento capaz de favorecer al enfermero en la prevención y tratamiento de las lesiones por presión.

Palabras clave: Lesión por presión; Estudio de validación; Cuidado de enfermera; Protocolos; Enfermería.

\section{Introdução}

Em 2016, na conferência de consenso realizada em Chicago, EUA, foi redefinido o termo Úlcera por Pressão, e passou a ser chamada de Lesão por Pressão (LPP). A LPP é caracterizada pela lesão localizada na pele e/ou tecido subjacente, geralmente sobre uma proeminência óssea, ou relacionada a dispositivos médicos, em consequência da pressão ou combinação desta com o cisalhamento, considerando-se outros fatores que afetam a tolerância tecidual, como a nutrição, micro clima, perfusão, comorbidades e as condições do próprio tecido (NPUAP, 2016).

No Brasil foi instituído pelo Ministério da Saúde, o Programa Nacional de Segurança do Paciente (PNSP), por meio da publicação da Portaria GM n ${ }^{\circ}$. 529. A segurança do paciente visa à redução de danos desnecessários, envolvendo a prática de ações que minimizem as complicações ao contexto e aos dispositivos a partir dos quais se presta o cuidado. Uma das ações previstas está voltada à prevenção de lesões por pressão em serviços de saúde (Brasil, 2013).

A LPP corresponde ao terceiro tipo de evento mais frequentemente notificado pelo PNSP dos hospitais brasileiros. Segundo dados de 2014 do Sistema de Notificações da Vigilância Sanitária (NOTIVISA), das 8435 notificações de evento adverso relacionado à assistência à saúde, 1319 foram notificações de LPP. (Vocci, Toso \& Fontes, 2017).

No Brasil, em Unidades de Terapia Intensiva (UTI), as taxas de incidência de lesões por pressão são altas e variam entre 13,95\% em São Paulo a 59,5\% em Fortaleza, dependendo da especialidade da UTI e da região do país. Em clínica médica, cirúrgica, ortopédica e UTI de três hospitais a taxa de incidência variou entre 25\% e 66,6\% no Mato Grosso (Baron et al., 2017). Outro estudo realizado em UTI no Rio de Janeiro apresentou 26,83\% para a taxa de incidência de LPP. (Park, Choi \& Kang, 2015).

As taxas de incidência e prevalência de LPP são consideradas como um indicativo da qualidade do cuidado de enfermagem. De acordo com a American Nursing Association (ANA), quando se desenvolvem após a admissão hospitalar, essas lesões agregam um valor negativo à qualidade da assistência (Costa et al., 2015).

O cuidado de pacientes com LPP pode ser bastante desafiador para os enfermeiros, tornando evidente a necessidade de que conheçam profundamente as características, os sinais e sintomas, fatores de risco, tipos de tratamentos, efeitos colaterais e os cuidados de enfermagem que podem ser prestados (Santos \& Gravitol, 2016).

Neste contexto, de organização das ações de cuidado de enfermagem, a adoção da tecnologia educativa, na prática permite uma maior relação entre o profissional e o paciente, pois o enfermeiro poderá utilizar o material no esclarecimento de dúvidas, além de propiciar o enfrentamento diante de alguma dificuldade manifestada (Dodt et al., 2015).

O uso de tecnologias educativas impressas, como cartilhas utilizadas nas práticas de educação em saúde, tem a proposta de oferecer informação sobre promoção da saúde, prevenção de doenças, modalidades de tratamento e autocuidado, apresentando-se como um instrumento facilitador do processo educativo (Siddharthan et al., 2016).

Cartilhas são materiais educativos capazes de promover resultados expressivos para os participantes das atividades educativas. Estudos enfatizam a importância da utilização de materiais educativos, como o desenvolvimento de uma cartilha educativa com o foco na promoção da saúde de mulheres grávidas (Reberte, Hoga \& Gomes, 2012), e a elaboração de uma cartilha educativa para portadores de diabetes mellitus, evidenciando que o uso possibilitou melhor compreensão da doença por parte do paciente e família (Meinert, Marcon \& Oliveira, 2011).

Diante destas considerações, este estudo teve como objetivo descrever o processo de construção e validação de uma a cartilha educativa para enfermeiros sobre prevenção e tratamento das lesões por pressão. A proposta é construir uma cartilha constituída de diretivas para enfermeiros, no formato de material impresso e digital, que possibilite a tomada de decisão acerca 
das melhores práticas assistenciais e gerenciais de cuidado, com a incorporação de saberes e práticas evidenciadas na literatura no direcionamento, padronização e sistematização das ações de cuidado quanto à prevenção e tratamento da lesão por pressão, aplicado como instrumento de promoção da saúde e facilitador do processo educativo da equipe de enfermagem (Santos \& Gravitol, 2016).

\section{Metodologia}

\section{Tipo de estudo}

Trata-se de estudo com abordagem metodológica, desenvolvido em três etapas: 1. Revisão integrativa; 2. Elaboração do material educativo e 3. Validação do material por juízes especialistas no assunto e público-alvo, os enfermeiros.

\section{Etapas do estudo}

Na etapa 1 foi realizada uma revisão integrativa das orientações de prevenção e tratamento da LPP, com o objetivo de levantar os materiais e instrumentos publicados, ampliando o conhecimento do tema. Realizou-se em seis etapas, a saber: 1. Identificação do tema e formulação do problema de pesquisa; 2. Estabelecimento de critérios para inclusão e exclusão de estudos; 3. Definição das informações a serem extraídas dos estudos selecionados; 4. Avaliação dos estudos incluídos na revisão integrativa; 5. Interpretação dos resultados; 6. Síntese do conhecimento evidenciado nas produções analisadas (Silva \& Christovam, 2017). O problema de pesquisa foi: Quais conteúdos são abordados em tecnologias educacionais acerca das ações de prevenção e tratamento de lesão por pressão são evidenciados na literatura científica? Os descritores utilizados foram: Pressure Ulcer; Protocols; Bandages; Nursingcare. As bases de dados consultadas foram: Medical Literature Analysis and Retrieval System Online (MEDLINE); Literatura Latino-Americana e do Caribe de Ciências da Saúde (LILACS); Scientific Electronic Library Online (ScIELO); Base de Dados de Enfermagem da Biblioteca Virtual em Saúde (BDENF/BVS); Cochrane Library.

Para determinar os estudos a serem analisados foram estabelecidos critérios de inclusão: produções disponíveis nas bases de dados, que versassem sobre a temática acerca das ações de prevenção e tratamento em pacientes portadores e/ou com potencial de risco para o desenvolvimento de lesão por pressão. Optou-se por publicações disponíveis "online", com textos completos e em português, inglês e espanhol. Foram excluídos estudos em duplicidade.

Em seguida, foi realizada a seleção dos artigos, analisando seus títulos. Após procedeu-se à leitura dos resumos e seleção dos estudos que se adequavam aos eixos norteadores. Posteriormente, os artigos pré-selecionados foram submetidos à leitura e releitura na íntegra, e incluídos na revisão apenas os que, por concordância das pesquisadoras, atendiam aos objetivos do estudo.

Etapa 2 as pesquisadoras elaboraram os textos baseadas no resultado da revisão integrativa, abordando os cuidados para a prevenção e tratamento de lesão por pressão. O trabalho de design e diagramação das imagens foi realizado por profissional de propaganda e marketing. As imagens foram recolhidas da internet e, posteriormente, trabalhadas no Adobe Illustrator. A versão final da cartilha possui 30 páginas frente e verso.

Na etapa 3 para coleta de dados foi utilizado um formulário autoaplicável adaptado (Rodrigues et al., 2013), dividido em duas partes: a primeira com dados de identificação do juiz e a segunda, contém instruções de preenchimento e 40 itens distribuídos em sete aspectos avaliativos, sendo dois de conteúdo (Exatidão científica e Conteúdo) e os cinco de aparência (Apresentação literária; Ilustrações; Material suficientemente específico e compreensivo; Legibilidade e características da impressão e Qualidade da informação). 


\section{População do estudo}

Nessa etapa, a pesquisadora submeteu a cartilha a juízes considerados especialistas no conceito em estudo. Foram convidados nove juízes, sendo que oito aceitaram e participaram de todas as etapas do estudo (Joventino et al., 2013) por meio da amostragem intencional, que atendessem aos critérios pré-estabelecidos adaptados de Fehring (1994), como possuir tese/dissertação/especialização na área lesão cutânea, participação em grupos/projetos de pesquisa na área lesão cutânea, prática profissional na área lesão cutânea, trabalhos publicados e experiência da temática de validação de instrumento ou materiais educativos.

Os juízes foram convidados a participar do estudo através de uma Carta Convite, via correio eletrônico, a qual trouxe os objetivos da pesquisa. Em seguida, aqueles que aceitaram participar da pesquisa, receberam também por correio eletrônico, um kit composto por: 1. Termo de Consentimento Livre e Esclarecido (TCLE); 2. Instrumento de validação e 3. Cartilha educativa. Os juízes puderam realizar a avaliação da cartilha no próprio domicílio, ou em outro local que lhe fosse mais conveniente, sendo estabelecido um prazo de quinze dias para que o mesmo realizasse a análise, preenchesse o instrumento de avaliação e os devolvessem à pesquisadora via correio eletrônico.

Após a realização dos ajustes necessários na cartilha das sugestões dos juízes especialistas, ocorreu a validação da aparência pelo público-alvo, ou seja, pelos enfermeiros de um Hospital Municipal em Niterói/RJ. A validação da cartilha junto aos indivíduos que vivenciam o tema abordado é necessária, já que são o foco da atividade educativa que se pretende realizar. Trata-se de um momento em que se possibilita verificar o que não foi compreendido, o que deve ser acrescentado ou aperfeiçoado (Guimarães et al., 2015).

Foram considerados elegíveis para o estudo, todos os enfermeiros do hospital independente do turno e regime de trabalho. Foram excluídos da amostra os afastados do serviço por licença médica ou férias nos períodos que os dados foram coletados e que não aceitaram participar de pelo menos uma das etapas. Assim, foram convidados 56 enfermeiros, dos quais 17 não retornaram o contato, o que resultou em uma amostra final de 39 enfermeiros que assinaram o TCLE e que participaram de todas as etapas da pesquisa.

Inicialmente foram convidados, pessoalmente, pela pesquisadora. Houve a leitura do Termo de Consentimento Livre e Esclarecido (TCLE) pelos enfermeiros junto à pesquisadora, a fim de sanar quaisquer dúvidas que pudessem surgir. Em seguida a cartilha foi apresentada aos enfermeiros do plantão reunidos em uma única sala, e por fim, o instrumento de coleta de dados para avaliação da aparência da cartilha foi autoaplicável. A duração da coleta de dados foi em média de 20 a 30 minutos.

\section{Tratamento e análise de dados}

Para validação do conteúdo foi utilizado o Índice de Validade de Conteúdo (IVC). Esse método emprega a utilização de escala tipo Likert para avaliar a concordância e a representatividade dos itens com pontuação de um a quatro e baseia-se nas respostas dos juízes com relação ao grau de relevância de cada item, assim, poderiam ser classificados como: (1) Não relevante ou não representativo; (2) Necessita de grande revisão para ser representativo; (3) Necessita de pequena revisão para ser representativo; (4) Relevante ou representativo.

O escore do índice é calculado por meio da soma de concordância dos itens que foram marcados por " 3 " ou "4" pelos especialistas, dividida pelo número total de respostas. Os itens que receberam pontuação "1" ou "2" devem ser revisados ou eliminados. Preconizam um valor mínimo para o IVC de 0,78 . Neste estudo, por se tratar de um instrumento novo utilizamos a taxa de 0,80, como mínima de concordância (Coluci, Alexandre \& Milani, 2015).

Quanto a aparência da cartilha, foi calculada a porcentagem de escores obtidos no instrumento. Este cálculo foi realizado por meio do somatório total dos escores, dividido pelo total de itens do questionário. Na análise dos dados foram 
considerados validados os itens com nível de concordância mínima de 75\% nas respostas positivas (Coluci, Alexandre \& Milani, 2015).

\section{Aspectos Éticos}

Foram respeitados todos os aspectos éticos relacionados à pesquisa com seres humanos contidos na Resolução ${ }^{\circ}$ 466/12 do Conselho Nacional de Saúde, publicada no Diário Oficial da União em 13 de julho de 2013. O projeto teve aprovação do CEP sob n CMM/HUAP n ${ }^{\circ}$ 149/2010.

\section{Resultados}

Na etapa 1, as estratégias de buscas nas bases de dados a associação de descritores que apresentou melhor refino foi: Pressure ulcer and protocols and nursing care or bandages tendo sido a escolha para dar andamento à pesquisa, resultando em 143 publicações. A Figura 1 representa o fluxograma de seleção dos estudos.

Figura 1 - Fluxograma de seleção dos estudos.

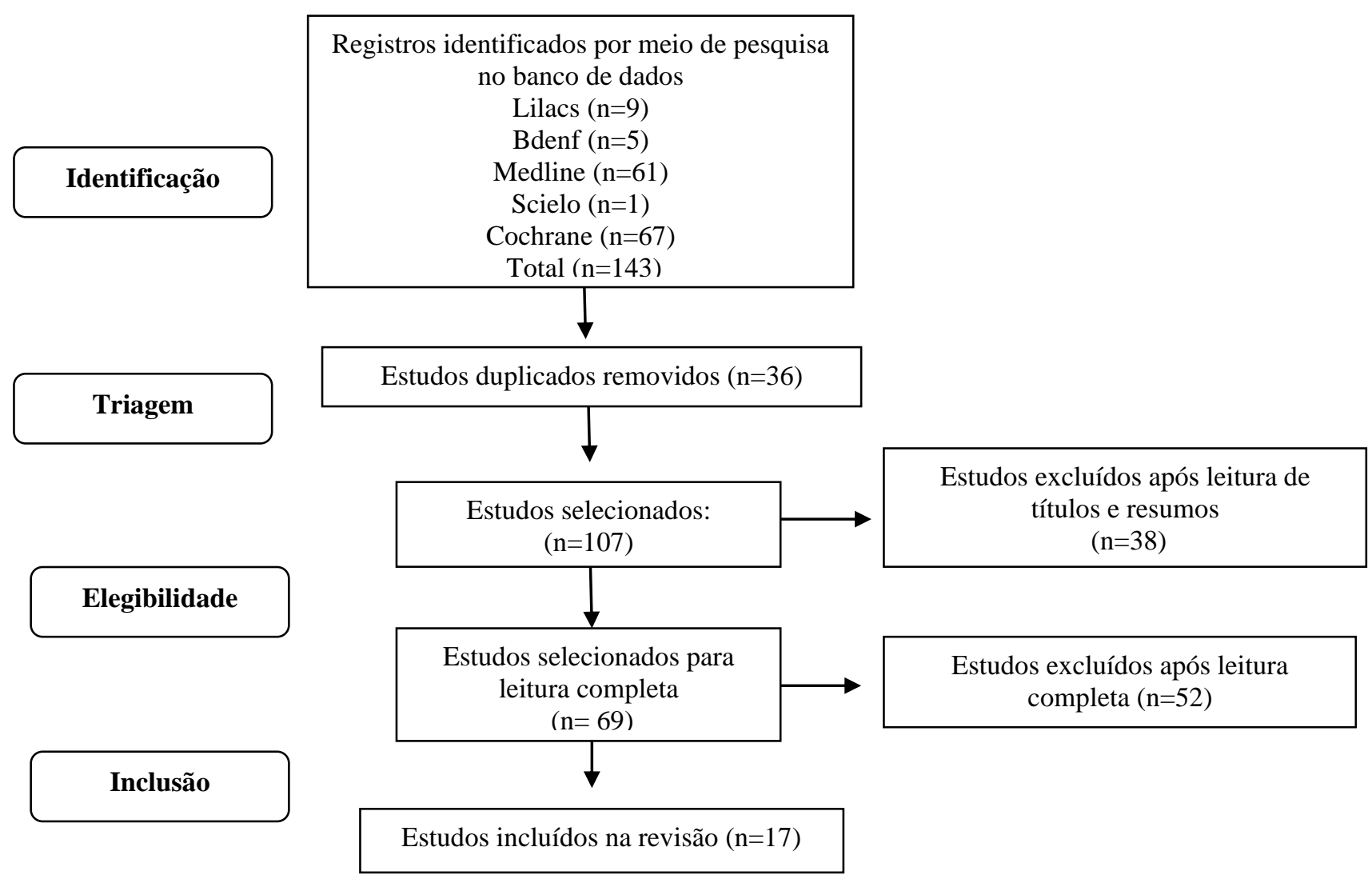

Fonte: Autores.

Sendo assim, utilizadas 17 publicações, todas relacionadas à lesão por pressão e/ou tecnologia educativa. São 10 artigos, 02 dissertações de mestrado e 05 protocolos estabelecidos pela National Pressure Ulcer Advisory Panel, European Pressure Ulcer Advisory Panel e Pan Pacific Pressure Injury Alliance, pelo Ministério da Saúde, pela prefeitura de Vila Velha e pelo Hospital de Anchieta/DF.

Na etapa 2, realizou-se a elaboração textual, seguido da confecção das ilustrações e finalizou-se com a diagramação, ficando com tamanho de papel A5 $(148 \times 210 \mathrm{~mm})$. A capa foi composta pelo título "Cartilha de orientações sobre prevenção e 
tratamento de lesão por pressão". Com contribuição da revisão de literatura a cartilha ficou dividida em doze domínios: Apresentação; Conceito da Lesão por pressão; Fatores de risco; Identificação e avaliação de risco; Classificação da lesão por pressão; Prevenção; Avaliação da lesão por pressão; Realização do curativo; Desbridamento; Coberturas indicadas; Critérios para um curativo ideal; Registro da lesão.

$\mathrm{Na}$ etapa 3, de validação da cartilha, foi avaliada por juízes especialistas e público-alvo. Foram selecionados oito juízes especialistas. O perfil destes é apresentado na Tabela 1.

Tabela 1 - Caracterização dos juízes especialistas, n=8. Niterói, 2018.

\begin{tabular}{ll}
\hline Variáveis & $\mathbf{n}(\%)$ \\
\hline Idade & $2(25,0)$ \\
22 a 31 anos & $4(50,0)$ \\
32 a 47 anos & $2(25,0)$ \\
48 a 63 anos & \\
Tempo de Formado & $2(25,0)$ \\
1 - 5 anos & $2(25,0)$ \\
6 - 10 anos & $3(37,5)$ \\
11 - 20 anos & $1(12,5)$ \\
$>21$ anos & \\
Titulação & $4(50,0)$ \\
Doutorado & $2(25,0)$ \\
Mestrado & $2(25,0)$ \\
Especialização em Estomaterapia & \\
Área de Atuação & \\
Docência & $2(25,0)$ \\
Assistência em estomaterapia & $2(25,0)$ \\
Comissão de curativo & $4(50,0)$ \\
\hline
\end{tabular}

Fonte: Autores.

Quanto os critérios pré-determinados, era necessária uma pontuação mínima de 5 pontos, que foram alcançados (Tabela 2), sendo a média 17,7 pontos. Esse fato revela maior confiança depositada nos especialistas, constatando experiência destes na temática de interesse.

Tabela 2 - Caracterização dos juízes de acordo com os critérios de seleção, Niterói, 2018.

\begin{tabular}{lcc}
\hline \multicolumn{1}{c}{ CRITÉRIOS } & N & $\%$ \\
\hline Tese/dissertação/especialização na área de interesse*(2 pontos/trabalho) & 8 & 100,0 \\
Participação em grupos/projetos de pesquisa na área de interesse*(1ponto/ano) & 6 & 75,0 \\
Prática profissional na área de interesse* (2 pontos/ano) & 8 & 100,0 \\
Trabalhos publicados (1 pontos/trabalho) & 5 & 62,5 \\
Experiência da temática de validação de instrumento ou materiais educativos *(2 pontos/ano) & 4 & 50,0 \\
\hline
\end{tabular}

Legenda: *Área de interesse: lesão cutânea. Fonte: adaptado de Fehring (1994).

No processo de validação da cartilha quanto ao conteúdo a "Exatidão Científica” obteve IVC igual a 0,93. Enquanto o “Conteúdo" obteve IVC de 0,96, indicando ótimo nível de concordância entre os especialistas. O IVC global da cartilha foi de 0,90, considerando-se, a cartilha validada quanto ao conteúdo. As sugestões dos enfermeiros foram incluídas e a cartilha foi revisada. 
Quanto à validação da aparência pelos juízes, constatou-se um valor de concordância global de 95,8\% para os cinco aspectos avaliativos do instrumento, variando de 84,3 a 100\%, níveis superiores ao mínimo estabelecido de $75 \%$, o que valida a cartilha também quanto a aparência.

Porém alguns especialistas mesmo avaliando bem os itens, fizeram sugestões para melhoria da cartilha tanto em relação a aparência quanto ao conteúdo e incluíram propostas de acréscimos de informações, que foram acatadas (Quadro 1).

Quadro 1 - Modificações realizadas na cartilha a partir das sugestões dos juízes.

\begin{tabular}{|ll|}
\hline Sugestões dos juízes & Modificações realizadas \\
\hline Reformulação de ilustração & Reformulada figura da capa \\
Substituição de termos técnicos & Substituída "fibrina" por "desvitalizado" \\
Reformulação de frase & Reformulação "aplicar a cobertura escolhida (calçar luva estéril)" por "calçar \\
luva estéril; aplicar a cobertura escolhida" & $\begin{array}{l}\text { Acrescentado Coberturas: } \\
\text { Estágio I : Silicone; }\end{array}$ \\
& Tecido de granulação: AGE, Hidrogel, Polihexanida Biguanida (PHMB), \\
& $\begin{array}{l}\text { Petrolatum; } \\
\text { Tecido Desvitalizado/ Necrosado: Colagenase, Polihexanida Biguanida }\end{array}$ \\
& (PHMB); \\
& Sinais de infecção e Odor Fétido: Alginato com Prata, Espumas de Prata. \\
\hline
\end{tabular}

Fonte: Autores.

Quanto à caracterização dos representantes do público-alvo, 39 enfermeiros, com idade entre 32-47 anos. Em relação à titulação nove tinham especialização, sendo duas em estomaterapia, e nenhum com doutorado ou mestrado. Com relação a área de atuação no hospital, quinze eram da Emergência, dezesseis da clínica médica, cinco do UTI, e três atuavam no setor de SIDA.

Os enfermeiros responderam o instrumento de validação da aparência da cartilha, assim pôde-se verificar o nível de concordância para os cinco aspectos avaliativos do instrumento (Figura 2). 
Figura 2 - Nível de concordância entre o público-alvo dos aspectos avaliativos da validação de aparência.

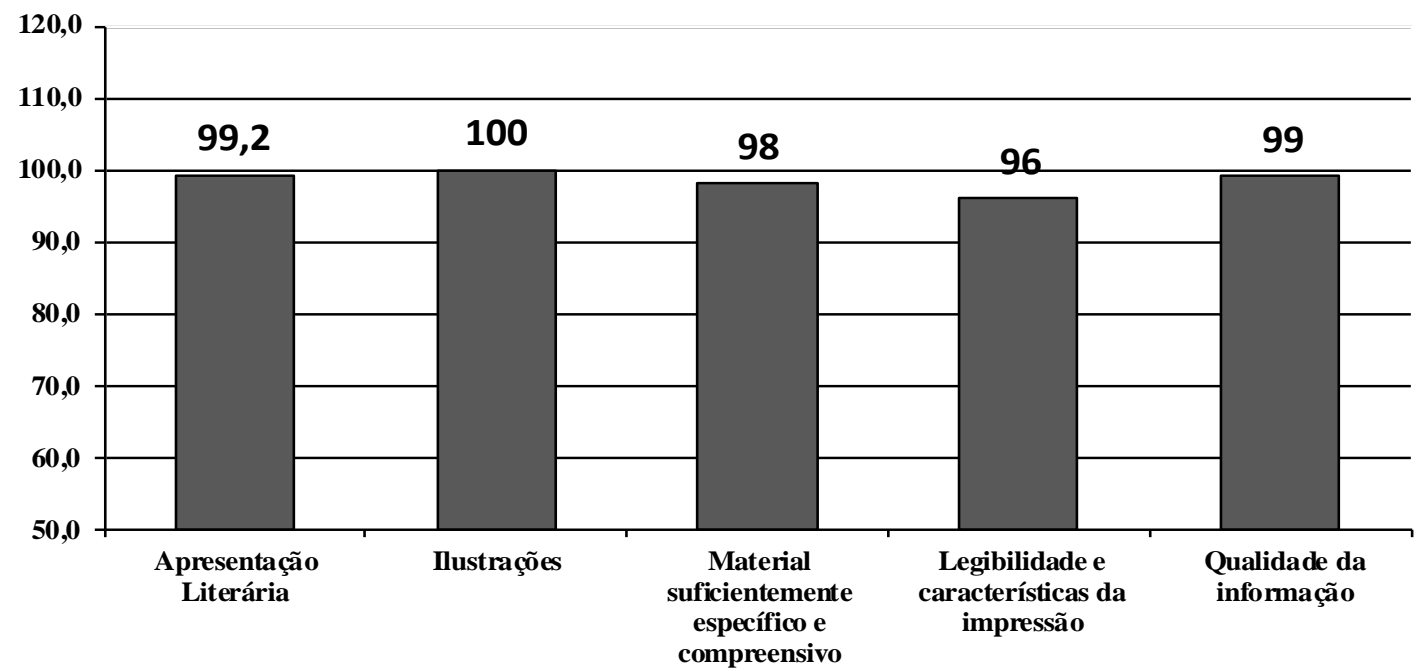

Fonte: Autores.

De acordo com a Figura 1, os percentuais de concordância entre os blocos evidenciaram valores de $96 \%$ a $100 \%$. Nesse aspecto, deduz-se que a cartilha alcançou o grau de significância de validação quanto à aparência também pelo públicoalvo.

Da amostra total, $100 \%$ concordaram com a aplicabilidade do material educativo para a prática clínica do enfermeiro. Os enfermeiros julgaram a cartilha relevante para ajudar nas orientações durante a assistência de enfermagem. Os enfermeiros foram solicitados, ao final da avaliação, a emitirem suas opiniões pessoais acerca da cartilha em geral. O Quadro 2 mostra uma síntese desse resultado.

Quadro 2. Opiniões do público alvo sobre a cartilha.

\begin{tabular}{|c|c|}
\hline Opiniões do público-alvo & \\
\hline Clareza & $\begin{array}{l}\text { "Gostei de tudo que visualizei, leitura clara e objetiva, bem explicado, não é cansativa, } \\
\text { muito interessante" }\end{array}$ \\
\hline \multirow[t]{2}{*}{ Conteúdo } & "Cartilha tem um conteúdo que explica de uma forma correta" \\
\hline & $\begin{array}{l}\text { "Gostei de toda a cartilha e conteúdo, tudo perfeito, clara e objetiva, não precisa revisar } \\
\text { conteúdo" }\end{array}$ \\
\hline \multirow[t]{2}{*}{ Didática } & "Cartilha didática" \\
\hline & "Gostei da dinâmica" \\
\hline \multirow[t]{3}{*}{ Promoção do conhecimento } & $\begin{array}{l}\text { "Gostei muito do trabalho e acredito que ele possa vir a somar com a assistência de } \\
\text { enfermagem em todo o hospital" }\end{array}$ \\
\hline & $\begin{array}{l}\text { "Gostei da explicação de cada material a ser utilizado conforme necessidades. } \\
\text { Totalmente válida, com ótima instrução" }\end{array}$ \\
\hline & $\begin{array}{l}\text { "Gostei muito nos ajuda com os conceitos e informações técnicas de orientar as ações } \\
\text { ao cuidado a ser realizado pelos enfermeiros" }\end{array}$ \\
\hline Ilustrações da cartilha & "Figuras claras e explicativas \\
\hline
\end{tabular}

Fonte: Autores. 


\section{Discussão}

A tecnologia educacional em saúde não é implementar apenas ações burocráticas, assistenciais, tarefeiras, pontuais e isoladas, mas, sobretudo é a maneira sistemática de organizar e planejar o processo de ensino-aprendizagem em termos de objetivos e da combinação de recursos humanos e materiais para resolver os problemas da saúde. Considera-se a tecnologia educacional como metodologia inovadora que possibilita dar ênfase ao processo de ensino-aprendizagem e avaliação, ao proporcionar protagonismo aos profissionais de saúde, colocando-os como agentes proativos e estimulando-os a buscar respostas para problemas reais e complexos com autonomia, tornando-os corresponsáveis na tomada de decisão (Schwonke et al., 2011).

Dentre os profissionais de saúde envolvidos no uso de ações de caráter educativo, os enfermeiros são constantemente desafiados a buscar opções que lhes ofereçam suporte para atuarem junto às pessoas, aos grupos e às comunidades, tendo as tecnologias educativas como fortes aliadas nesse processo. Todavia, para que esses profissionais utilizem essa ferramenta de maneira eficaz, é preciso que elas sejam desenvolvidas e validadas (Berardinell et al., 2014).

$\mathrm{O}$ uso de tecnologias educativas impressas, como cartilhas, são ferramentas úteis e importantes a serem utilizadas nesse processo de ensino-apredizagem, alternativa viável para informação e sensibilização dos profissionais de saúde, podendo abrir caminhos novos para a promoção da saúde, além de permitir ao profissional de saúde uma leitura posterior, que reforça orientações verbais, servindo como guia em casos de dúvidas e auxiliando as tomadas de decisão cotidianas (Lacerda et al., 2011).

A cartilha educativa surgiu a partir do conhecimento ainda limitado dos enfermeiros frente a prevenção e tratamento das lesões por pressão, elevando as taxas de incidência e prevalência, indicando-se a necessidade de produções que potencializem esta modalidade de assistência, e foi construída obedecendo a critérios científicos, sendo portanto, uma estratégia com potencial de reunir conhecimentos capazes de subsidiar um cuidado integral, seguro e de qualidade (Galvão et al, 2017)

Os dados da pesquisa contribuem para divulgação de informações importantes preconizadas pelo protocolo de lesão por pressão, integrante do Programa Nacional de Segurança do Paciente, do Ministério da Saúde (Brasil, 2013). Assim, a partir da diagramação do conteúdo em caixas de textos e tópicos e com ilustrações do texto, o material fica mais fácil para ser compreendido durante a leitura (Silva, Bezerra \& Brasileiro, 2017).

O vocabulário utilizado em materiais impressos e/ou digitais deve ser coerente com a mensagem que se pretende transmitir e com o público-alvo a que se destina. Espera-se que sua leitura seja fácil, convidativa e compreensível (Silva, Bezerra \& Brasileiro, 2017). A fim de garantir a legibilidade e a compreensão do texto os seguintes achados de pesquisa foram: emprego de palavras curtas, conhecidas e de formação simples; repetição de palavras importantes; articulação de frases; uso de voz ativa; apresentação de conceitos e ações em ordem lógica (Guimarães et al., 2015).

Pesquisas antropológicas destacam que a agudeza visual é um veículo de comunicação que dispõe de grande influência sobre os valores sociais, morais e éticos, constituindo um código totalmente aceito e compreendido. Somente se os cartazes, cartilhas, manuais, folhetos e folders, impressos e/ou digitais, estiverem totalmente integrados ao imaginário social é que alcançarão seu propósito, veiculando, por símbolos e signos, as mensagens que se quer passar para o público-alvo (Stothard et al., 2016).

A viabilidade de utilização de cartilhas educativas perpassa pela compreensão do leitor acerca do conteúdo que é apresentado no material. Resultados de estudo, realizado na Suécia, mostram que $29 \%$ dos materiais educativos fornecidos em 27 hospitais aos pacientes submetidos à cirurgia de câncer colorretal eram de difícil compreensão e os autores apontam que a investigação da opinião do público-alvo pode contribuir para a obtenção de materiais educativos mais adequados à linguagem do leitor. Em relação à compreensão de cartilha educativa, estudo realizado no leste da África, que avaliou o impacto da 
educação em saúde na escola sobre a esquistossomose, aponta que 75\% dos leitores não compreendiam informações contidas em cartilha educativa (Teles et al., 2014).

A validação da cartilha junto aos indivíduos que vivenciam o tema nele abordado, o público-alvo, é uma atitude necessária, já que os mesmos são o foco da atividade educativa que se pretende realizar. Trata-se de um momento de suma importância, em que se possibilita verificar o que não foi compreendido, o que deve ser acrescentado ou aperfeiçoado, além de se perceber a distância entre o que foi exposto e o que foi apreendido (Stothard et al., 2016).

Nessa perspectiva, os juízes especialistas que realizaram a validação de conteúdo sugeriram pequenos ajustes relacionados à adequação de linguagem em termos técnicos e na gramática da cartilha em frases que poderiam dificultar a interpretação dos enfermeiros. Esse processo de adaptação do material educativo às sugestões é uma etapa essencial para tornar a tecnologia mais completa, de maior rigor científico e eficaz durante a atividade de educação em saúde. Essa etapa é referida também por outros estudos como de grande relevância para aperfeiçoamento do material a ser validado, nos quais, da mesma forma, foram sugeridas a reformulação e a exclusão de informações, substituição de termos, além da reformulação das ilustrações (Reberte, Hoga \& Gomes, 2012).

A aplicação da tecnologia educativa de prevenção das lesões por pressão deve conter informações relativas à identificação do cliente, escala de avaliação de risco para desenvolvimento de lesão por pressão, quadro demonstrativo das áreas suscetíveis às lesões, registro das modificações da pele, seguindo os estágios das lesões por pressão e guia de prevenção (Silva, Bezerra \& Brasileiro, 2017).

A efetividade do tratamento das lesões por pressão depende essencialmente da utilização de coberturas adequadas (Silva, Bezerra \& Brasileiro, 2017), contribuindo para o processo de cicatrização, a partir desta afirmação os juízes propuseram um leque maior de coberturas baseado no estágio desta lesão, o tipo de tecido, a quantidade de exsudato, a presença de odor e infecção.

Em tecnologias educativas, a utilização de ilustrações consiste em uma importante ferramenta para o processo de comunicação. No entanto, depender da forma como a ilustração é apresentada, pode contribuir positiva ou negativamente para o processo educativo (Teles et al., 2014).

Assim, o presente estudo contribui para o avanço científico na ciência da enfermagem ao disponibilizar, para o meio assistencial e acadêmico, cartilha com enfoque educativo que foi validada por juízes e avaliado pelo público-alvo podendo ser utilizada na prática clínica de forma impressa ou digital.

\section{Considerações Finais}

A cartilha foi validada, sendo considerada no contexto das atividades educativas como um instrumento capaz de favorecer as medidas de prevenção e tratamento da lesão por pressão, por meio de linguagem e ilustrações claras, objetivas, acessíveis e atraentes aos enfermeiros.

Acredita-se que o uso deste material facilitará a prática dos enfermeiros, tendo em vista que se constitui em uma tecnologia ilustrada capaz de facilitar a aquisição de conhecimentos por parte destas, memorização dos cuidados necessários a prevenção e tratamento das lesões por pressão, proporcionar o empoderamento dos enfermeiros, bem como um meio de padronizar as orientações.

Como limitação desse estudo, tem-se a não validação por especialista técnico em comunicação. Tendo-se concluído a construção e validação da cartilha, o estudo não termina aqui, ressalta-se que a cartilha passará por atualizações contínuas mediante o progresso científico e tem-se a intenção de levar o material validado para uso nos serviços especializados e de se realizar pesquisas futuras para avaliar o custo-efetividade e sua eficácia no alcance da implementação das medidas de prevenção e tratamento da lesão por pressão. 
A tecnologia foi licenciada com a Licença Creative Commons - CC BY-NC-SA e depositada no Repositório recebendo o seguinte identificador: http://educapes.capes,gov.br/handle/capes/573186.

\section{Referências}

Agência Nacional de Vigilancia Sanitária - Anvisa. Resolução da Diretoria Colegiada da Anvisa - RDC n 36 , de 25 de julho de 2013 . Institui ações para a segurança do paciente em serviços de saúde e dá outras providências. Diário Oficial da União

Baron, M. V., Pavani, R. M., \& Forgiarini, J. L. A. (2017) Innovations and technologies for the prevention of pressure ulcers in the calcaneus. Revista Epidemiologia e Controle de Infecções, 7(2),122-131.

Berardinell, L. M., Guesdes, N. A., Ramos, J. P., \& Silva, M. G. (2014) Tecnologia educacional como estratégia de empoderamento de pessoas com enfermidades crônicas. Rev Enferm UERJ, 22(5),603-609

Coluci, Z. O., Alexandre, M. C., \& Milani, D. N. M. (2015) Construção de instrumentos de medida na área da saúde. Ciência \& Saúde Coletiva, 20(3),925936.

Costa, A. C. O., Pinho, C. P. S., Santos, A. D. A. D., \& Nascimento, A. C. S. D. (2015) Úlcera por presión: incidencia y factores demográficos, clínicos y nutricionales asociados en pacientes de una unidad de cuidados intensivos. Nutrición hospitalaria, 32(5),2242-2252.

Dodt, R. C. M., et al. (2015) Estudo experimental de uma intervenção educativa para promover a auto eficácia materna na amamentação. Revista LatinoAmericana de Enfermagem, 23(4), 725-732.

Fehring, R. J., (1994) The Fehring Model. In: Carrol-Johnson, R. M., Paquete, M., editors. Classification of nursing diagnoses: Proceedings of the Tenth Conference: JB Lippincott, 55-62.

Galvão, N. S., et al. (2017) Conhecimentos da equipe de enfermagem sobre prevenção de úlceras por pressão. Revista Brasileira de Enfermagem, 70(2),312318.

Guimarães, F. A. B., et al. (2015) Avaliação de material didático elaborado para orientação de cuidadores e professores de creches sobre o desenvolvimento infantil. Journal of Human Growthand Development, 25(1), 27-40.

Joventino, E. S., Oriá, M. O., Sawada, N. O., \& Ximenes, L. B. (2013) Apparent and content validation of maternal self-efficiency scale for prevention of childhood diarrhea. Rev Lat Am Enfermagem, 21(1),371-379.

Lacerda, R. A., Nunes, B. K., Batista, A. O., Egry, E. Y., Graziano, K. U., Ângelo, M., et al. (2011) Evidence-based practices published in Brazil: identification and analysis of their types and methodological approaches. Rev Esc Enferm USP, 45(3),773-782.

Meinert, L., Marcon, C., \& Oliveira, L. D. B. (2011) Elaboração de cartilha educativa para paciente diabético como intervenção psicológica: um trabalho multiprofissional. Psicologia para América Latina, 22, 1-14.

National Pressure Ulcer Advisory Panel. National Pressure Ulcer Advisory Panel (NPUAP) (2016) Announces a change in terminology from pressure ulcer to pressure injury and updates the stages of pressure injury.

Park, S., Choi, Y., \& Kang, C. (2015) Predictive validity of the Braden Scale for pressure ulcer risk in hospitalized patients. Journal of Tissue Viability, 24(3),102-113.

Reberte, L. M., Hoga, L. A. K., \& Gomes, A. L. Z. (2012) O processo de construção de material educativo para a promoção da saúde da gestante. Rev. LatinoAm. Enfermagem, 20(1), 101-108

Rodrigues, A. P., Nascimento, L. A. D., Dodt, R. C. M., Oriá, M. O. B., \& Ximenes, L. B. (2013) Validation of a flipchart for promotion of self-efficacy in breastfeeding. Acta Paulista de Enfermagem, 26(6), 586-593.

Santos, E. I., \& Grativol, A. D. O. J. (2016) Social representations of nurses about professional autonomy and the use of technologies in the care of patients with wounds. Investigacion y educacion en enfermeria, 34(2), 378-386.

Schwonke, C. R. G. B., Filho, W. D. L., Lunardi, V. L., Santos, S. S. C., \& Barlem, E. L. D. (2011) Perspectivas filosóficas do uso da tecnologia no cuidado de enfermagem em terapia intensiva. Rev bras enferm, 64(1), 189-192.

Silva, H. L., Bezerra, F. H. G., \& Brasileiro, L. C. (2017) Avaliação de materiais educativos direcionados para o desenvolvimento neuropsicomotor da criança. Revista Brasileira em Promoção da Saúde, 30(3), 1-6.

Siddharthan, T., Rabin, T., Canavan, M. E., Nassali, F., Kirchhoff, P., Kalyesubula, R., et al. (2016) Implementation of patient-centered education for chronicdisease management in Uganda: an effectiveness study. PLoS One, 11(11), e0166411.

Silva, T. S. G., \& Christovam, B. P. (2017) Sistema de indicadores como ferramenta de monitoramento e avaliação das ações da gerência de cuidado de enfermagem- um estudo metodológico. Rio de Janeiro, 2017. (Dissertação em Enfermagem) Universidade Federal Fluminense, Rio de Janeiro.

Stothard, J. R., Khamis, A. N., Khamis, I. S., Neo, C. H. E., Wei, I., \& Rollinson, D. (2016) Health education and the control of urogenital schistosomiasis: assessing the impact of the juma na kichocho comic-strip medical booklet in Zanzibar. J Biosoc Sci, 48(Suppl 1), S40-55.

Teles, L. M. R., et al. (2014) Construção e validação de manual educativo para acompanhantes durante o trabalho de parto e parto. Revista da Escola de Enfermagem da USP, 48(6), 977-984. 
Research, Society and Development, v. 10, n. 3, e3810312926, 2021

(CC BY 4.0) | ISSN 2525-3409 | DOI: http://dx.doi.org/10.33448/rsd-v10i3.12926

Vocci, M. C., Toso, L. A. R., \& Fontes, C. M. B. (2017) Application of the Braden Q scale at a pediatric intensive care unit. J Nurs UFPE on line, 11(1), 165172. 